\title{
A thyrotoxicosis outbreak due to dietary pills in Paris
}

\author{
Vincent loos' \\ Vincent Das' \\ Eric Maury ${ }^{1,2}$ \\ Jean-Luc Baudel' \\ Jérôme Guéchot ${ }^{3}$ \\ Bertrand Guidet ${ }^{1,2}$ \\ Georges Offenstadt ${ }^{1,2}$ \\ 'Réanimation Médicale; ${ }^{2}$ Université \\ Pierre et Marie Curie-Paris 6, \\ INSERM, UMR-S 707; ${ }^{3}$ Unité \\ d'Hormonologie, APHP, Hôpital Saint \\ Antoine, F-750 I2, Paris, France
}

Correspondence: Eric Maury

Service de Réanimation Médicale, Hôpital

Saint-Antoine, Assistance Publique-

Hôpitaux de Paris, 184 rue du Faubourg

Saint-Antoine, 7557I Cedex I2, Paris,

France

Tel +33492823 I8

Fax +3349282145

Email eric.maury@sat.aphp.fr

\begin{abstract}
Three women were consecutively admitted to our medical intensive care unit for thyrotoxicosis after the ingestion of dietary pills accidentally containing high levels of thyroxin. These cases were observed during an outbreak in the Paris area. Despite similar blood levels of thyroid hormones, their clinical presentation and outcome were very different. One patient developed febrile confusion and died from malignant hyperthermia. The second one had progressive confusion requiring mechanical plasma exchange therapy and had a favorable outcome. The third one had very moderate symptoms. These exceptional observations raise several issues concerning diagnosis, physiopathology and treatment of thyrotoxicosis factitia.
\end{abstract}

Keywords: thyrotoxicosis, dietary pills, thyroxin

\section{Patient I}

A 57-year-old woman was admitted to our Medical Intensive Care Unit (MICU) for shock, coma and high fever. She had no relevant medical history but was taking spironolactone, metformin, doxylamine and "homeopathic pills". Her weight was $77 \mathrm{~kg}$ and height $176 \mathrm{~cm}$. Ten days before admission, the patient reported headache. Three days before admission in our MICU, she became confused. Next day, she was referred to the emergency department of another hospital (hour 0, H0). She was disorientated and agitated without neurological abnormalities. Blood pressure was 190/90 $\mathrm{mmHg}$, pulse, 146 beats per minute and temperature, $38^{\circ} \mathrm{C}$. Brain CT scan and CSF analysis were normal. She was given $100 \mathrm{mg}$ of intravenous loxipamine $(\mathrm{H} 2)$, aciclovir, ceftriaxone and amoxicillin and was admitted to the medical ward. As cultures of blood, urine and CSF were negative, and the C-reactive protein value was normal, anti-infective therapies were discontinued. However, the temperature remained high $\left(39.2^{\circ} \mathrm{C}, \mathrm{H} 24\right)$ despite acetaminophen. Because of increasing agitation she was given $100 \mathrm{mg}$ of intravenous tiapride at $\mathrm{H} 14$ and H32. Her consciousness afterwards deteriorated (Glasgow Coma Scale GCS $=4$ ) and she became hypotensive. She received fluid loading (normal saline), norepinephrine, and the trachea was intubated. Whole body CT scan showed no abnormalities. The patient was then referred to our MICU (H40). She had severe circulatory failure, with diffuse mottling, oliguria and a mean blood pressure of $70 \mathrm{mmHg}$ while receiving $1.5 \mu \mathrm{g} / \mathrm{kg} / \mathrm{min}$ norepinephrine. Central body temperature was $40.9{ }^{\circ} \mathrm{C}$ (Figure 1). Room temperature was $20^{\circ} \mathrm{C}$. Transthoracic echocardiography showed a hyperkinetic heart but no other abnormality. On admission, blood test results were: sodium: $154 \mathrm{mmol} / \mathrm{L}$, bicarbonates: $16 \mathrm{mmol} / \mathrm{L}$, chloride: $125 \mathrm{mmol} / \mathrm{L}$, lactates: $3 \mathrm{mmol} / \mathrm{L}$, creatine kinase: $588 \mathrm{IU} / \mathrm{L}$, creatininaemia $76 \mu \mathrm{mol} / \mathrm{L}$. Blood gas analysis showed $\mathrm{pH}: 7.33, \mathrm{PaO}_{2}: 229 \mathrm{mmHg}$ while under a $75 \%$ inspired fraction of oxygen and PaCO2: $32 \mathrm{mmHg}$. Total blood count was normal, prothrombin time, $47 \%$, coagulation factor $\mathrm{V}, 62 \%$ and fibrinogen, $6.2 \mathrm{~g} / \mathrm{L}$. Serum toxic screening with mass spectrography was negative. Hydrocortisone $(100 \mathrm{mg}$ 3 times a day) was started (a later report showed that cortisol level was normal). 


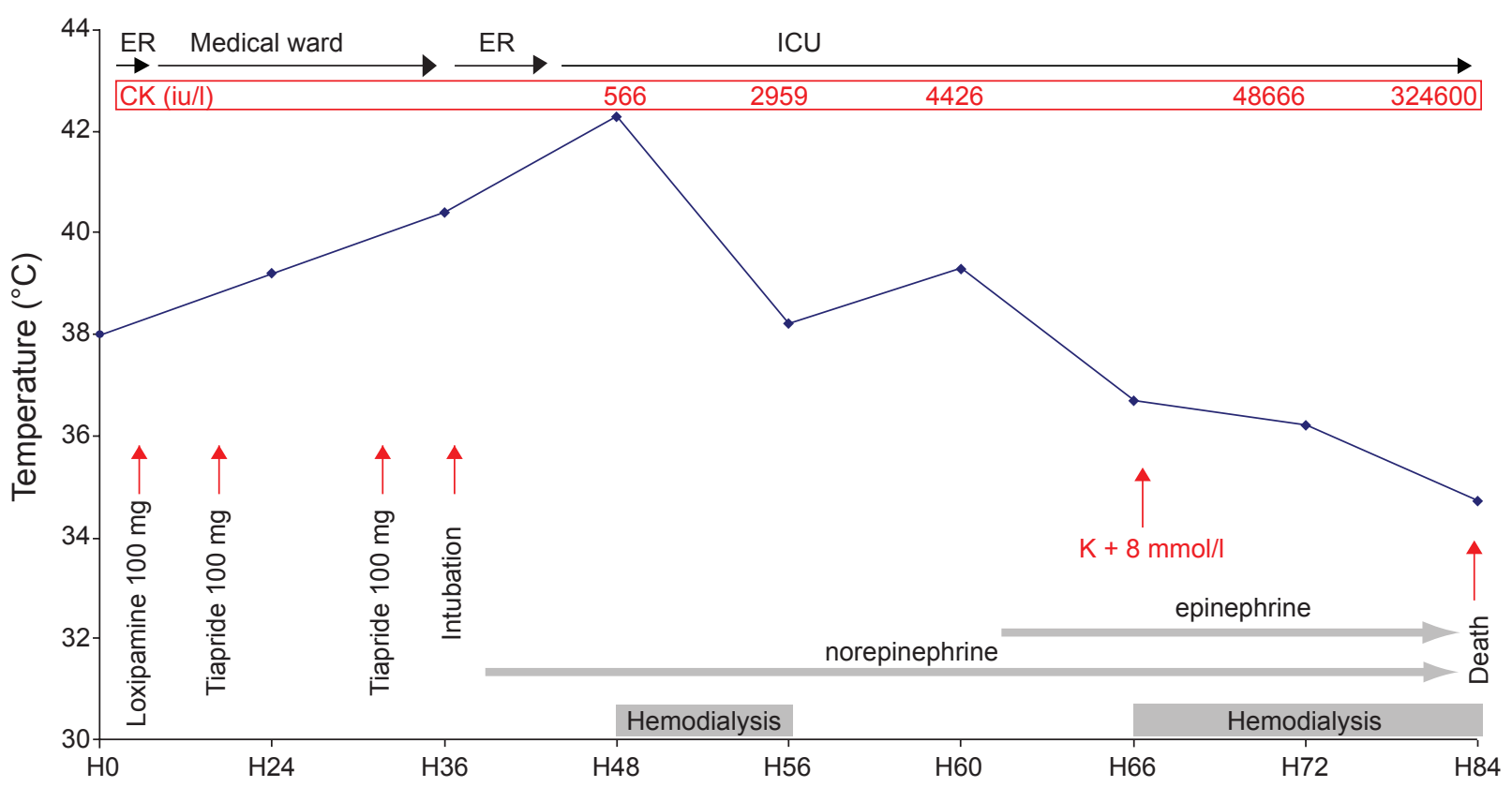

Figure I Time course and events of the evolution of Patient one during her stay in the first hospital (admission in the Emergency Room [ER] and referral to the ward) and in our hospital (admission in the ER and then in Intensive Care Unit (ICU]). Red arrows indicate main events, grey areas represent sustained therapy. The blue diagram depicts temperature evolution. The plasma creatine phosphokinase (CPK) are in red at the top of the figure.

Although there was no documented bacterial infection, vancomycin, cefotaxime and gentamicin were started. Because the patient's body temperature rose to $42.3{ }^{\circ} \mathrm{C}$ at $\mathrm{H} 48$ despite external cooling, hemodialysis was performed with a dialysate temperature of $33^{\circ} \mathrm{C}$. There was no muscular rigidity. The patient's temperature went down to $38.2{ }^{\circ} \mathrm{C}$ by the end of dialysis (H56). However, circulatory failure worsened and epinephrine was administered because of left ventricle hypokinesia (H62). Hemodialysis was again started because of symptomatic hyperkaliemia $(8 \mathrm{mmol} / \mathrm{L}$, H66) related to massive rhabdomyolysis. The patient died of refractory shock, 43 hours after MICU admission (H84). Two days later, the French health authorities issued a message warning against the toxicity of "dietary pills", which had been manually prepared in one particular pharmacy and contained huge amount of thyroxin. It turned out that these "dietary pills" were the "homeopathic pills" taken by our patient. Analysis of blood drawn on MICU admission confirmed hyperthyroidism (Table 1).

\section{Patient 2}

A 71-year-old previously healthy woman was admitted to our MICU 5 days after admission of Patient one for severe thyrotoxicosis. She had a history of depression and had been treated for 1 year with similar "dietary pills". Her free T4 and TSH levels were normal in 6 months before. One week before admission, she reported fatigue, headache, myalgia, palpitations and fever. She stopped the dietary pills. The day of admission, mild confusion occurred. The patient was contacted by health authorities as a patient who had been prescribed the suspectedly toxic "dietary pills". She was referred to our institution (day 0, D0) because of high levels of serum thyroid hormone (Table 1). The patient was awake but severely confused. She had no chills, sweating, jaundice or neck rigidity. Blood pressure was 130/60 and body temperature was $37{ }^{\circ} \mathrm{C}$. Except for tachyarrhythmia, cardio-pulmonary examination was normal. ECG revealed atrial fibrillation. The patient was given $40 \mathrm{mg}$ oral propranolol 4 times a day, $100 \mathrm{mg}$ hydrocortisone 3 times a day, cholestyramine 3 times a day, and was given 4 tablets of propylthiouracyl 4 times a day for 24 hours and subcutaneous heparin. On D3, she was unable to speak and severely agitated. No muscular rigidity was observed. Because of clinical degradation and high plasma thyroid hormone levels, plasma exchange (PE) therapy was performed on D3 with 1.51 of $4 \%$ albumin and $1.5 \mathrm{~L}$ of hydroxy-ethyl-amidon, and repeated on D4 with the addition of $1 \mathrm{~L}$ fresh frozen plasma (Figure 2). After a second PE, the patient was placed on a ventilator because of impaired consciousness (GCS: 7). An EEG did not reveal any features of epilepsy. A CT scan of the head was normal. The thyroid hormone levels slowly returned to close to normal, the patient's consciousness gradually improved. She was extubated on D10. She experienced a perfect cognitive recovery but exhibited flaccid tetraparesia. 
Table I Levels of blood thyroid hormones in the three patients on admission to intensive care unit

\begin{tabular}{lllll}
\hline Thyroid hormone & normal & patient I & patient 2 & patient 3 \\
\hline Total T4 $(\mathrm{pmol} / \mathrm{L})$ & {$[80-160]$} & 404 & 469 & 631 \\
Free T4 $(\mathrm{pmol} / \mathrm{L})$ & {$[7.5-21.1]$} & $>75$ & $>75$ & $>75$ \\
Free T3 $(\mathrm{pmol} / \mathrm{L})$ & {$[3.6-7]$} & $>45$ & $>45$ & $>45$ \\
TSH $(\mathrm{mU} / \mathrm{L})$ & {$[0.15-3.7]$} & 0 & 0 & 0.02 \\
\hline
\end{tabular}

An electromyogram confirmed myoneurogen abnormalities. On D25 she was referred to a rehabilitation facility for persistent inability to stand up and walk alone.

\section{Patient 3}

A 40-year-old woman was admitted to our MICU 5 days after the admission of Patient two (day 0, D0) for thyrotoxicosis. Her medical history was uneventful. She had been overweight (94 kg, $165 \mathrm{~cm}$ ), and had taken the "dietary pills", since 2004. Three weeks before admission, she started complaining of asthenia, myalgia, headache, sweating, tremor of the lower limbs and dyspnea. She spontaneously stopped the pills 6 days before admission. She heard about the toxicity of the pills through the media and went to the emergency department of our hospital. On admission, physical examination was normal except sinusal tachycardia (113 beats/min). Echocardiography and EEG were unremarkable. She was admitted to the MICU for monitoring and was treated with $40 \mathrm{mg}$ oral propranolol 4 times a day, cholestyramine 3 times a day and $100 \mathrm{mg}$ intravenous hydrocortisone 3 times a day. The thyroid hormone levels measured at D1 showed hyperthyroidism (Table 1). On the same day the patient was agitated and was given $100 \mathrm{mg}$ oral clorazepate. She improved and was referred to the endocrinology department on D4. She had then an uneventful follow-up. At D10, her thyroid hormone levels were still high : TSH $=0.03 \mathrm{mIU} / \mathrm{L}(0.15-3.7)$, total $\mathrm{T} 4=264 \mathrm{pmol} / \mathrm{L}(80-160)$, free T4 = $66.5 \mathrm{pmol} / \mathrm{L}(7.5-21.1)$ and free $\mathrm{T} 3=9 \mathrm{pmol} / \mathrm{L}(3.6-7)$.

\section{Methods}

Written informed consent to publication of the present reports was obtained from each patient or from their closest relatives. Thyroid tests were performed using the following methods: TSH, Access US hTSH (Beckman Coulter), normal range : 0.15-3.7 mIU/L; Unbound tri-iodothyronine, Access Free T3 (Beckman Coulter), normal range : 3.6-7.0 pmol/L; Unbound Thyroxin, Access Free T4 (Beckman Coulter), normal range: 7.5-21.0 pmol/L; Total Thyroxin, Access

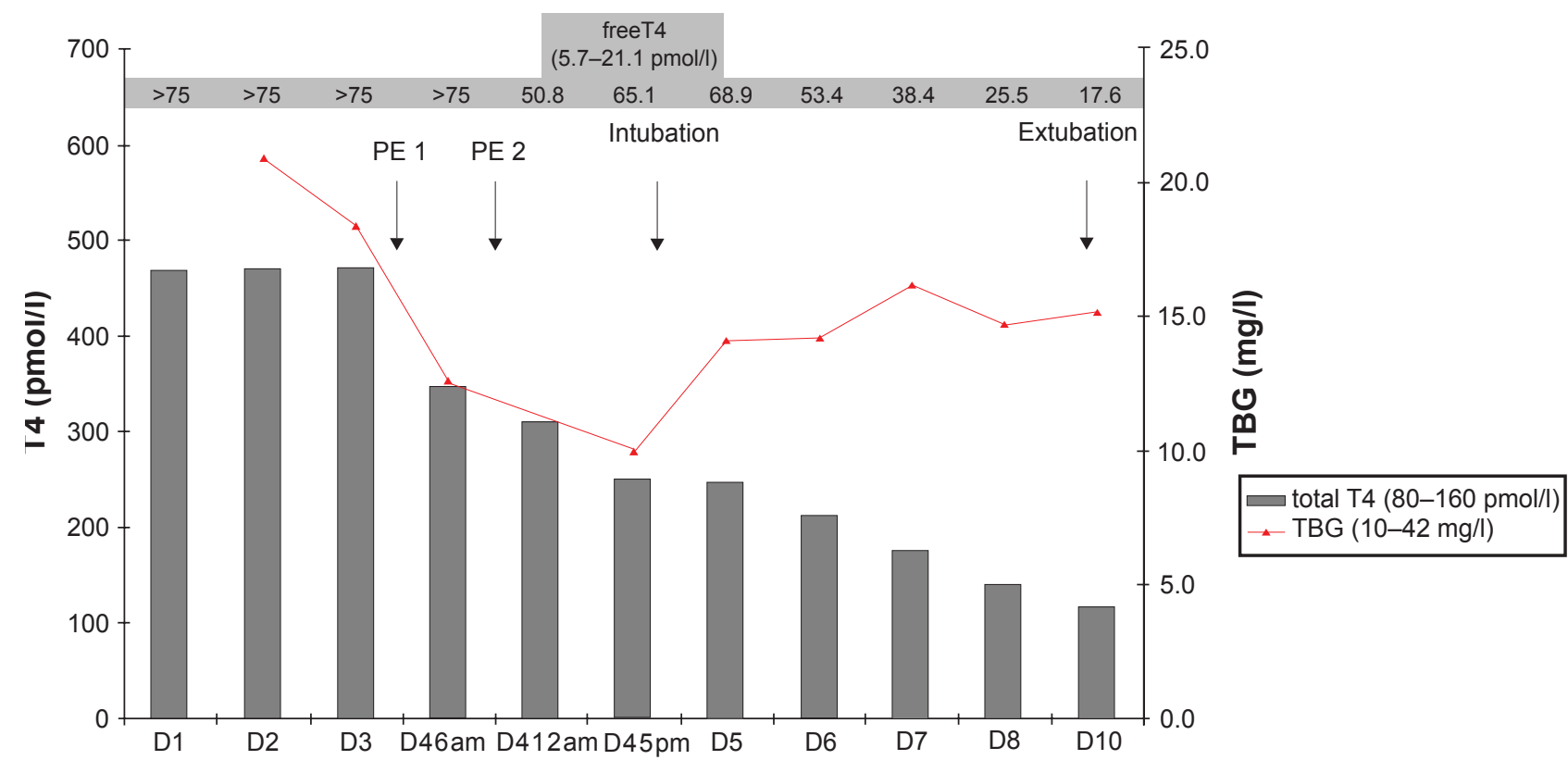

Figure 2 Serum thyroxin (T4) and thyroxine-binding globulin (TBG) before and after plasma exchange (PE) therapy in Patient 2. Total plasma T4 evolution is represented by vertical grey bars, free T4 evolution is written in the grey area at the top of the diagram. 
total T4 (Beckman Coulter) normal range: 75-160 mol/L; Thyroid-Binding Globulin, RIA-gnost TBG (CISbio International), normal range (non-pregnant adults): 10-42 mg/L.

\section{Discussion}

Thyrotoxicosis factitia is the intentional or inadvertent ingestion of thyroid hormones in excess. It has been described as a complication of an inappropriate substitutive therapy, in the course of a non indicated L-thyroxin treatment for obesity (Yoon et al 2003), following the ingestion of beef contaminated with thyroid gland (Parmar and Sturge 2003) and after accidental ingestion (Bhasin et al 1981). Treatment of thyrotoxicosis facticia should include the discontinuation of intoxication, gastric lavage in the case of acute ingestion, $\beta$ blockers, digoxin or hydrocortisone to counteract the action of thyroid hormones and/or reduce the conversion of T4 to T3. There is no need for treatments aimed at suppressing endogenous thyroid hormones production such as neomercazole, radioiodine, or thyroidectomy. Plasma exchange therapy is advocated for life-threatening conditions (Binimelis et al 1987; Kokuho et al 2004), because it has been shown to remove $25 \%$ to $30 \%$ of the hormones ingested. Sodium ipodate, used for oral cholecystography, has been recommended because it inhibits the type I iodothyronine 5'-monodeiodinase which catalyses the T4 to T3 conversion.

Our cases can be classified as both a complication of treatment for obesity and as accidental thyroxine ingestion. The "dietary pills" were ordered by the same practitioner for the treatment of obesity, prepared by the same pharmacy and labelled "phytotherapy-homeopathy". Each pill usually contained thyroid compounds, caffeine, carrageenan, sibutramine, and infra-therapeutic doses of L-thyroxine $(25 \mu \mathrm{g})$, diazepam, aminophylline and metformin. All three patients had taken these pills for months or years. A toxicological investigation revealed that $30 \mathrm{mg}$ of L-thyroxin had been put into each of the pills responsible for the poisoning. In addition to our patients, 34 other women were identified as having taken those pills sold during 4 days. Fifteen of them were admitted to hospital and 9 of them to ICU. None of these patients died. An outbreak of thyrotoxicosis due to errors in the preparation of pharmaceutical agents was reported 20 years ago in Spain among patients on substitutive therapy for thyroid disease (Binimelis et al 1987).

Despite a fulminant presentation, thyroid storm was not diagnosed in patient 1 . Her practitioner's prescription was illegible, and the patient's family reported that she was taking "homeopathy" to lose weight. The initial encephalitis-like initial presentation followed by multi-organ failure made hyperthyroidism difficult to diagnose. The skin was neither moist nor warm. No tremor or sweating were noted. Although these signs are usually reported during thyroid storm, they may be absent in cases of severe dehydration. Confusion and occasionally coma have been observed in thyroid storm (Newmark et al 1974; Howton 1988; Tietgens and Leinung 1995), but their association with high fever is first of all suggestive of an infectious disease. This implies that in case of a critically ill patient said to be on weight reduction, the possibility of thyroid hormone ingestion should be considered (Braunstein et al 1986). Rhabdomyolysis has been observed during thyroid crisis (Bennett and Huston 1984; Hosojima et al 1992) but it never reached the magnitude observed in patient 1. Hyperthermia is common during thyroid storm (Simon 1986; Tietgens and Leinung 1995). Nevertheless extreme fever (more than $42^{\circ} \mathrm{C}$ ) associated with rhabdomyolysis is much more suggestive of heat stroke or malignant hyperthermia (Caroff and Mann 1993). Combined with the increased heat production due to hyperthyroidism, the neuroleptic agents might have enhanced the failure of thermoregulation. Several reports of malignant hyperthermialike pictures in patients with hyperthyroidism receiving general anesthesia (Murray 1978; Stevens 1983; Bennett and Wainwright 1989) support this hypothesis. We can not finally exclude that the final evolution of Patient one was precipitated by neuroleptic administration.

In patient 2, PE therapy was performed because of persistent confusion and cardiac arrhythmia. The level of thyroxine in the extracted plasma was close to the one measured in the serum (352 $\mathrm{pmol} / \mathrm{L}$ and $259 \mathrm{pmol} / \mathrm{L}$ respectively). Whereas the total T4 level decreased after the second PE, the free T4 level rose concomitantly, and a clinical deterioration was observed. The level of plasma thyoxine-binding globulin (TBG) decreased after second PE, therefore raising, at least theoretically, the fraction of free thyroxine in the plasma. Fresh frozen plasma could be used as a substitute during $\mathrm{PE}$, in order to supply thyroxin carriers and prevent any rise in free thyroxine levels, since $70 \%$ of thyroxine is bound to $\mathrm{TBG}$, and $20 \%$, to albumin. The clinical degradation after the second $\mathrm{PE}$ could have resulted from a rise in the free thyroxine level. However, clinical status has been reported to deteriorate between the 7 th and 10th days after ingestion, while serum hormone levels are decreasing (Meurisse et al 2000). This might reflect that clinical status is more closely correlated to the effects of thyroid hormones at the cellular level, than at their circulating levels. This is in accordance with the evolution of patient 3 , who had the highest level 
of total thyroxine with only minor symptoms. The absence of link between the thyroid hormones levels and clinical symptoms has been reported (Nystrom et al 1980).

Patients 2 and 3 also received $\beta$ blockers, hydrocortisone and cholestyramine (supposed to reduce thyroid hormones intestinal absorption ). Patient 2 was also given propylthiouracil on the first day for its inhibition effect on T4 to T3 conversion. Sodium ipodate is not available in France.

These observation suggest that thyroid hormone ingestion has to be suspected in any patient treated for obesity presenting with acute symptoms, especially confusion. Management should be based on the clinical symptoms and not on circulating hormone levels. When plasma exchange is considered, fresh frozen plasma should be used for substitution.

\section{Disclosures}

The authors have no conflicts of interest to disclose.

\section{References}

Bennett MH, Wainwright AP. 1989. Acute thyroid crisis on induction of anaesthesia. Anaesthesia, 44:28-30.

Bennett WR, Huston DP. 1984. Rhabdomyolysis in thyroid storm. Am J Med, 77:733-5.

Bhasin S, Wallace W, Lawrence JB, et al. 1981. Sudden death associated with thyroid hormone abuse. Am J Med, 71:887-90.
Binimelis J, Bassas L, Marruecos L, et al. 1987. Massive thyroxine intoxication: evaluation of plasma extraction. Intensive Care Med, 3:33-8.

Braunstein GD, Koblin R, Sugawara M, et al. 1986. Unintentional thyrotoxicosis factitia due to a diet pill. West J Med, 145:388-91.

Caroff SN, Mann SC. 1993. Neuroleptic malignant syndrome. Med Clin North Am, 77:185-202.

Hosojima H, Iwasaki R, Miyauchi E, et al. 1992. Rhabdomyolysis accompanying thyroid crisis: an autopsy case report. Intern Med, 31:1233-5.

Howton JC. 1988. Thyroid storm presenting as coma. Ann Emerg Med, 17:343-5.

Kokuho T, Kuji T, Yasuda G, et al. 2004. Thyroid storm-induced multiple organ failure relieved quickly by plasma exchange therapy. Ther Apher Dial, 8:347-9.

Meurisse M, Gollogly L, Degauque C, et al. 2000. Iatrogenic thyrotoxicosis: causal circumstances, pathophysiology, and principles of treatmentreview of the literature. World J Surg, 24:1377-85.

Murray JF. 1978. Hyperpyrexia of uncertain origin. Br J Anaesth, $50: 387-8$.

Newmark SR, Himathongkam T, Shane JM. 1974. Hyperthyroid crisis. JAMA, 230:592-3.

Nystrom E, Lindstedt G, Lundberg PA. 1980. Minor signs and symptoms of toxicity in a young woman in spite of massive thyroxine ingestion. Acta Med Scand, 207:135-6.

Parmar MS, Sturge C. 2003. Recurrent hamburger thyrotoxicosis. CMAJ, 169:415-7.

Simon HB. Extreme pyrexia. 1986. Hosp Pract (Off Ed), 21:123-4, 7-9.

Stevens JJ. 1983. A case of thyrotoxic crisis that mimicked malignant hyperthermia. Anesthesiology, 59:263.

Tietgens ST, Leinung MC. 1995. Thyroid storm. Med Clin North Am, 79:169-84.

Yoon SJ, Kim DM, Kim JU, et al. 2003. A case of thyroid storm due to thyrotoxicosis factitia. Yonsei Med J, 44:351-4. 
